\title{
Analysis of Breakdowns in Menu-Based Interaction Based on Information Scent Model
}

\author{
Yukio Horiguchi, Hiroaki Nakanishi, Tetsuo Sawaragi, and Yuji Kuroda \\ Graduate School of Engineering, Kyoto University, \\ Yoshida Honmachi, Sakyo-ku, Kyoto 606-8501, Japan \\ \{horiguchi, nakanishi, sawaragi\}@me.kyoto-u.ac.jp
}

\begin{abstract}
High communicability of the menu-based system is on the basis of consistent vision and clear policy in designing the system of menus, and then they should be perceivable to the users. In this light, failures in menu-based interactions can be explained that they might emerge from lack of information in the users' available cues to identify the design vision. This study focuses on communicative breakdowns in menu-based human-computer interactions from this perspective, and investigates their causes in ill-organized structures of menu hierarchy in terms of the user's interpretation of the menu items. Pirolli's information scent model is extended and utilized as an analytical tool for describing the meaning system of menus from the users' point of view, and their decision making in search of particular menu items is analyzed by use of information scent.
\end{abstract}

Keywords: Menu-based interaction, information scent model, communicative breakdowns, human-computer interaction.

\section{Introduction}

Menu design not compatible with the users' style of decision making easily misdirects and confuses their search of target items. When effects of a menu selection disagree with what was meant to be the case, it will disturb the user's understanding of how he/she may or must interact with the computerized system. This picture can be explained in terms that the user's mental model differs far from the design model or the designer's conceptual model of how the system should work [1], which means the user does not accommodate the proper usage of the system the designer originally intended. It is ideal that both of the mental models do coincide with one another in essentials. However, the user makes use of only a portion of the product functions in usual and will not necessarily experience all of them. Therefore, deliberate design of menus is required so that certain experiences with use of the system can enable the user to predict how he can access other unknown functions the system is equipped with. In other words, underlying design intent and principles should be communicated to the user through interactions with the system efficiently and effectively. de Souza emphasizes such an important role of interactive artifacts to mediate a kind of "designer-to-user communications", and redefine the concept of communicability in this sense [2]. 
Usability of a hierarchical menu system is characterized by a structure in which the menu items are organized as well as by a familiar terminology with which they are written, and both of these characters should be designed as sensible, comprehensible and convenient forms relevant to the user's task [3]. On the other hand, high communicability of the menu-based system is, by definition, on the basis of consistent vision and clear policy in designing such systems of menus, and then they should be perceivable to the users. In this light, failures in menu-based interactions can be explained that they might emerge from lack (or inconsistency) of information in the users' available cues to identify the designer's vision.

In this study, we focus on breakdowns in menu-based interactions from this perspective, and investigate their causes in ill-organized structures of menu hierarchy in terms of the user's interpretation of the menu items. Pirolli's information scent model [4-6] is extended and utilized as an analytical tool for describing the meaning system of menus from the users' point of view, and their decision making in search of particular menu items is analyzed by use of information scent. The scent measure, which can estimate the strength of each option to attract the user's attention relevant to a particular goal, is applied to specifications of possible discrepancies between the designer's intended usage and the user's actual decision.

\section{Information Scent of Menu Relevant to User's Goal}

Two different activation patterns derived from one common spreading activation network are compared for measuring the scent value of a menu item. One pattern of them represents the activities of concepts (to be precise, indexing words) induced by the user's goal whereas the other simulates the activities induced by the menu texts the user has encountered on the UI. The network of words was built from a text corpus, i.e., a large collection of documents, whose subject is to provide descriptions about the usage of the product's functions. In this network, every directed arc has weight derived from the conditional probability at which its source word would appear in a document containing its destination word, and each node has base level activation derived from the probability at which the corresponding word would appear in a document. So as to calculate these probabilities, we utilize instruction manuals as the corpus, which are decomposed into documents in accordance with its functional units, because it has sufficient statements about all the functions of the product in terms both of quality and quantity. The detailed descriptions on this calculation are given in [7].

As shown in Fig. 1, the scent value of a menu item is calculated according to the following procedure:

1. Each word's activation level induced by the user's goal, i.e., $\mathbf{L}=\left(L_{1}, L_{2}, \ldots\right)$, is derived after all words' activities in the user's task description $Q$ have spread in the network.

2. Each word's activation level induced by the menu texts, i.e., $\mathbf{R}=\left(R_{1}, R_{2}, \ldots\right)$, is derived after all words' activities in the target menu description $C$ have spread in the network.

3. The scent value of the menu item $C$ in relation to the task description $Q$ is given by the inverse Euclidean distance between the two activity patterns, i.e., $\mathbf{L}$ and $\mathbf{R}$. 
As is clear from its definition, the more similar the two activity patterns in response to the different activation sources, the larger the menu's information scent.

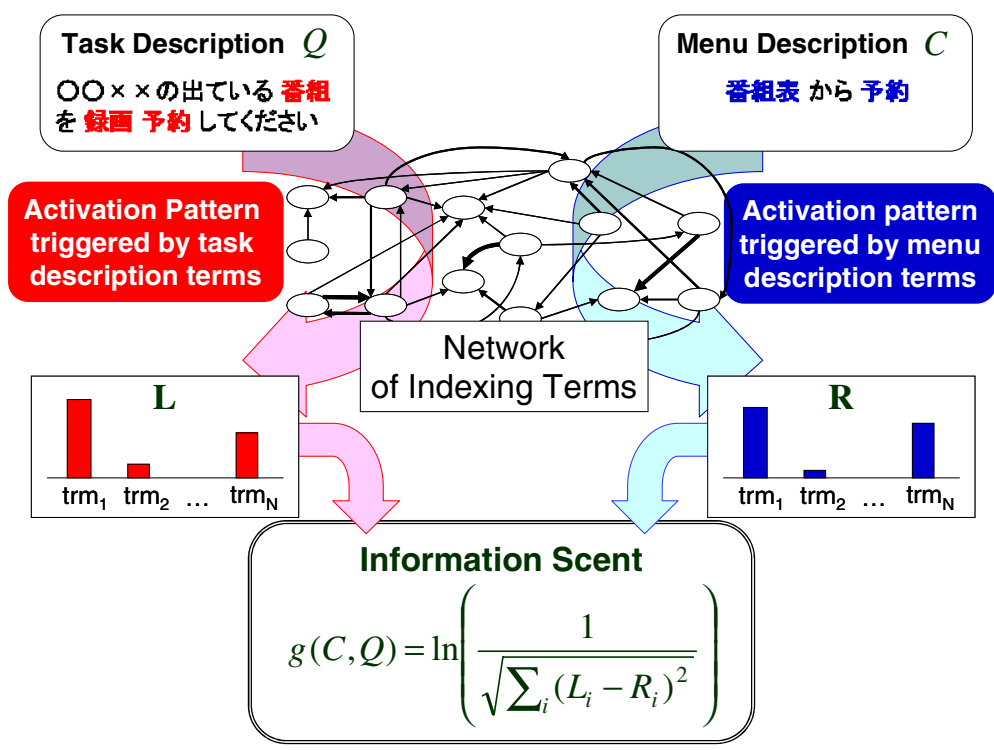

Fig. 1. Diagrammatic illustration of how the information scent of a menu item is calculated. Two different activation patterns derived from one common spreading activation network are compared for measuring the scent value of each menu item.

\section{Breakdowns in Menu-Based Interaction}

\subsection{Experiment}

A DVD recorder, which is one of typical multifunctional electric appliances that have hierarchical menus, was employed as the target application system. Twelve female users from three different age groups (30's, 40's and 50's: each age group contains four people) participated in the experiment, and all of them have no experiences with DVD recorders whereas they have some with VCRs. Four different tasks listed below were prepared for this experiment, and they are related to programming or configuring the recorder:

- Task 1: "Program the recorder for timer recording of a television show on which a particular on-screen talent will appear."

- Task 2: "This recorder has a capability to display closed captions on the television screen for terrestrial and BS digital broadcasts. Configure the recorder to display the captions."

- Task 3: "Configure the recorder for recording the second audio programs provided by multichannel broadcasting services." 
- Task 4: "This recorder has a capability to adjust timer recordings automatically to any airtime changes of the scheduled programs when some extension or delay of the prior programs has occurred. Configure the recorder for enabling this function."

The participants performed these tasks in the order from Task1 to Task4. Among them, the later task would be more difficult for the users because its goal is a peripheral function that is rare to be used and thus that is located at 'out-of-the-way' corners of the menu hierarchy.

Each task was specified on a sheet of paper $^{1}$ which was presented to the users immediately before a measurement session. After the experimenter had confirmed the user's sufficient understanding of the task without the sheet, he gave her a cue to start operation. During each session, the users were not allowed to refer to the sheets. A base time limit was set to four minutes that was used to judge the exit state of the participants' performances.

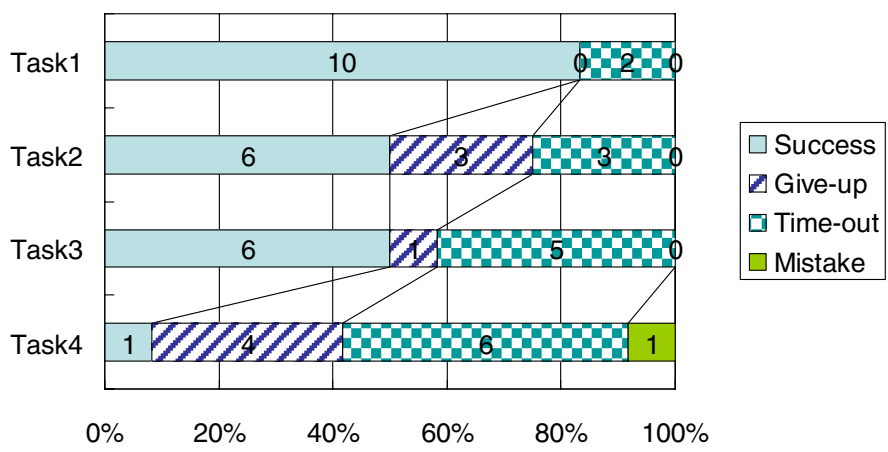

Fig. 2. Summary of the participants' performances. The results of individual sessions are classified into four different classes: "success", "give-up", "time-out" and "mistake".

\subsection{Results}

Fig. 2 presents the summary of the participants' performances where the results of their individual sessions are classified into four different classes: "success" represents the state that the user successfully completed her task in time; "give-up" represents to the state that the user gave up her task; "time-out" represents to the state that the user was interrupted by the experimenter to abandon her operations because it seemed to be no chance for her to complete the task; and "mistake" represents the state that the user could not find the correct menus although she declared she had finished the task for herself. The result indicates that Task 4 is the most difficult while Task1 is the easiest of all.

\subsection{Analysis of Failures during Menu-Based Interaction}

Low communicability of an interactive system can be evaluated by numerous patterns of slips, mistakes and failures spotted during interaction between the user and the system.

\footnotetext{
${ }^{1}$ The descriptions of all the tasks were given in Japanese.
} 
The concept of communicative breakdown is prepared to capture instances of such problematic interactions [2]. A communicative breakdown will appear during interaction between the user and the computerized system when the effects on the state of affairs induced by his/her operations do not coincide with what was meant to be the case. From this perspective, failures during menu-based interactions are analyzed here.

After all measurement sessions, the experimenter interviewed every participant about the reasons for her menu selections by watching playback videos together. With the use of their answers and comments as reference, failures of interactions between the users and the menu system were associated with the categories of communicative breakdowns. In accordance with de Souza's method [2], problematic portions of user-artifact interaction were tagged with one or more virtual "utterances" of the users corresponding to the categories of communicative breakdowns such as

- "What's this?" - the user is being unable to interpret what a certain interface element means,

- "Where is it?" — the user is not finding where his/her expected element is,

- "I can't do it this way." - the user is abandoning a path of interaction composed of many steps,

and so on. Fig. 3 illustrates an example of the analyzed discourse between a participant user and the DVD recorder where the user was performing Task1. In this figure, tags of communicative breakdowns are represented in the dialogue balloons.

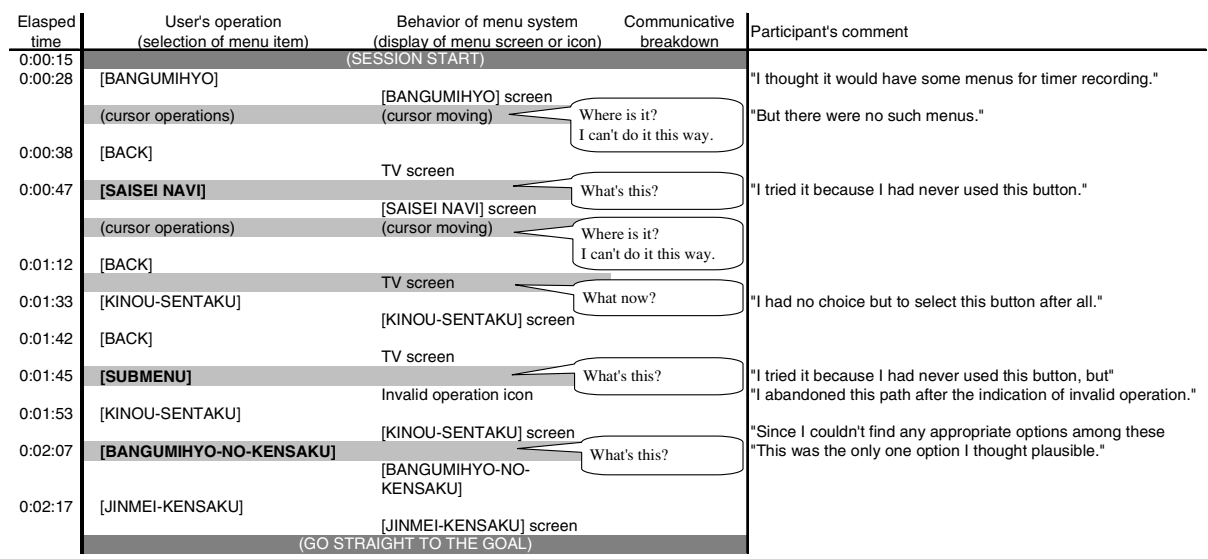

Fig. 3. An example of analyzed discourses where the user was performing Task1 with the use of the DVD recorder. The dialogue balloons represent the tags of communicative breakdowns.

As clarified in Fig. 2, Task1 and Taks4 have quite a difference in their success rate. Fig. 4 compares these two different tasks in terms of frequencies of the communicative breakdowns. This bar chart indicates that breakdowns tagged with "I can't do it this way." occurred in Taks4 more than twice as frequently as in Task1. This type of breakdown involves the user's becoming aware of a need for some reform of her search strategy since a series of her operations seemed not compatible with what the designer intended. Before it comes up in the user-system interaction, repetitions of "Where is 
it?" were observed when the user did not find a certain expected element (i.e., "it") among her selectable options. We can see a significant number of utterances of "Where is it?" in Task4 than those in Task1, and it reminds us that the design intent of the menu hierarchy should be distant from the users' assumptions for interpreting the interface signs. This hypothesis is also supported by the high frequency of "What's this?" because it corresponds to the breakdown where user is looking for any other cue about what a particular interface sign means. In addition, both of these breakdowns should induce another type of utterance "What now?". The latter indicates the situation where the user could not make sense of the interaction the designer intended and thus she was temporarily clueless about what to do next.

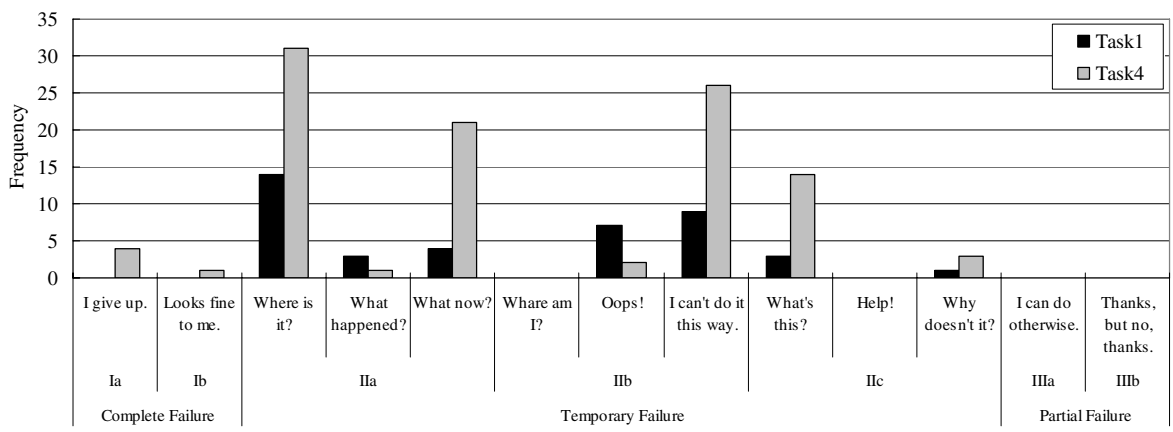

Fig. 4. Frequency distribution of communicative breakdowns. Task 1 and Taks 4 are compared in terms of frequencies of breakdowns because they have quite a difference in their success rate.

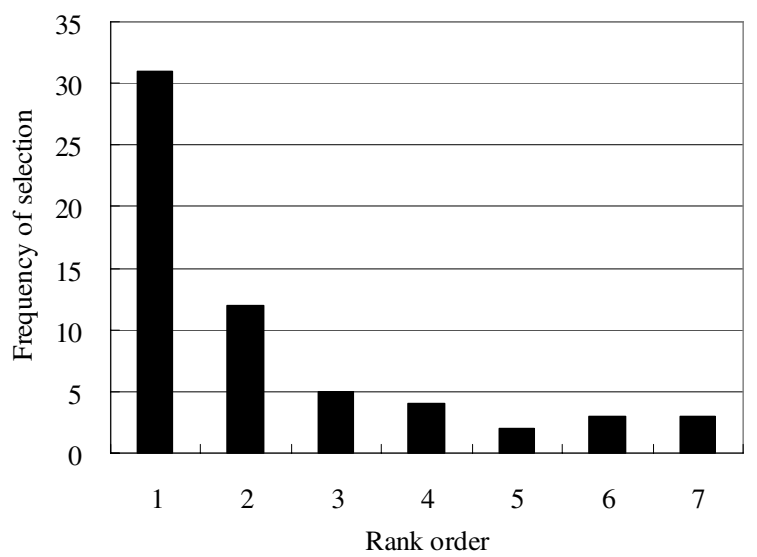

Fig. 5. Frequency distribution of the participant users' menu selections with respect to the rank order of information scent. The users selected menu items of higher rank order more frequently.

Both of the designer and the user have their distinctive assumptions for generating or interpreting the interface signs. The above result from the communication analysis suggests that there is a large difference between them, especially on peripheral functions 
like the goal of Task4. In order to visualize this difference, the information scent analysis is applied to the user's interaction with the menu system in the next section.

\section{Analysis of Breakdowns Based on Information Scent}

The decision strategy of the participant users can be explained by the perspective of information scent. Fig. 5 shows the frequency distribution of menu items that the users actually selected with respect to the rank order of information scent. The histogram illustrates that the higher rank order menu items have, the more frequently the users selected them. The scent distribution has thus a power to explain and predict the users' menu-selection behaviors.

On the basis of this finding, the organization of menus was analyzed through the scent distribution. Table 1 shows the scents of each menu in relation to the four different tasks, where the numbers in blue boldface represent the highest values of a menu list while the underlined numbers represent the correct options the users should select to get to the goals. Table 1(a) presents the scent distribution in the portal menu screen while Table 1(b) presents the distribution in the menu screen after MENU 7 is selected in this portal. These two tables show a significant tendency that the more successful tasks like Task1 have more manifest scent in their correct paths. Conversely, in the less successful tasks like Task4, menu options competing to the correct one have stronger scent toward the goals. These menus are not compatible with the users' decision strategy explained above. The analysis here indicates it can easily misdirect and confuse the users' search of the goal items by attracting their more attention. It is lack of information in the users' available cues. The users are in difficulty to identify how they may or must interact with this system, i.e., the design vision. This menu hierarchy can be said not to have a well-organized structure, especially for peripheral functions of the product.

Table 1. Scent distribution among menu items in two different menu screens. Scent values of individual menu items are listed with respect to each task.

(a) Portal menu screen

\begin{tabular}{|c|r|r|r|r|r|r|r|}
\hline & \multicolumn{1}{|c|}{ MENU 1 } & \multicolumn{1}{|c|}{ MENU 2 } & \multicolumn{1}{c|}{ MENU 3 } & \multicolumn{1}{|c|}{ MENU 4 } & \multicolumn{1}{c|}{ MENU 5 } & \multicolumn{1}{c|}{ MENU 6 } & \multicolumn{1}{c|}{ MENU 7 } \\
\hline Task1 & 1.168 & $\mathbf{2 . 7 0 4}$ & $\underline{2.135}$ & 1.331 & 1.373 & 1.139 & 0.976 \\
\hline Task2 & 0.898 & $\mathbf{1 . 5 3 9}$ & 1.530 & 1.260 & 1.304 & 0.839 & $\underline{1.292}$ \\
\hline Task3 & 1.100 & $\mathbf{1 . 5 3 5}$ & 1.511 & 1.218 & 1.283 & 1.032 & $\underline{1.305}$ \\
\hline Task4 & 1.052 & $\mathbf{2 . 1 3 7}$ & 2.106 & 1.478 & 1.564 & 1.062 & $\underline{1.218}$ \\
\hline
\end{tabular}

(b) 'MENU 7' screen

\begin{tabular}{|l|r|r|r|r|r|r|}
\hline & MENU 7-1 & MENU 7-2 & MENU 7-3 & MENU 7-4 & MENU 7-5 & MENU 7-6 \\
\hline Task1 & 1.048 & 2.513 & 0.983 & 1.179 & 1.078 & 1.175 \\
\hline Task2 & 0.825 & 1.066 & 1.099 & 1.671 & $\underline{\mathbf{2 . 0 3 6}}$ & 0.873 \\
\hline Task3 & 0.958 & 1.284 & 1.136 & $\underline{\mathbf{1 . 8 6 0}}$ & 1.695 & 1.069 \\
\hline Task4 & 0.951 & 1.528 & 1.074 & $\mathbf{1 . 7 9 7}$ & $\underline{1.659}$ & 1.059 \\
\hline
\end{tabular}




\section{Conclusion}

This paper discussed breakdowns in menu-based interactions between the users and the computerized system from the perspective of perceivable structures of the menu systems. Information scent model was utilized for comparing the meanings of menus from the users' point of view and then analyzing the users' decision makings in search of particular menu items.

The communicative breakdown analysis confirmed that there is a large difference between the designer and the users in assumptions for signifying or interpreting the menus (i.e., menu items and their organization for listing), especially in the case of the product's peripheral functions. On the other hand, the information scent analysis confirmed that the distribution of information scent among a menu list provides a powerful clue for predicting the user's menu selection. This result supports the findings that the success rate of the users' search will decrease if menu options competing to the correct one are designed to have stronger scents toward the goal. Menu designs not compatible with the users' naturalistic decision making can easily misdirect their search. The latter analysis specified the discrepancy between the designer and the users which was suggested in the former analysis.

\section{Acknowledgments}

This work has been partially supported by the Grant-in-Aid for Creative Scientific Research No.19GS0208 of the Ministry of Education, Culture, Sports, Science and Technology (MEXT) of Japan. We are grateful for their support.

\section{References}

1. Norman, D.A.: The Psychology of Everyday Things. Basic Books (1988)

2. de Souza, C.S.: The Semiotic Engineering of Human-Computer Interaction. MIT Press, Cambridge (2005)

3. Shneiderman, B.: Designing the User Interface: Strategies for Effective Human-Computer Interaction, 3rd edn. Addison-Wesley Longman, Amsterdam (1998)

4. Pirolli, P., Card, S.K.: Information Foraging. Psychological Review 106, 643-675 (1999)

5. Pirolli, P.: The Use of Proximal Information Scent to Forage for Distal Content on the World Wide Web. In: Kirlik, A. (ed.) Adaptive Perspectives on Human-Technology Interaction: Methods and Models for Cognitive Engineering and Human-Computer Interaction, pp. 247-266. Oxford University Press, Oxford (2006)

6. Pirolli, P.: Information Foraging Theory: Adaptive Interaction with Information. Oxford University Press, Oxford (2007)

7. Horiguchi, Y., et al.: Analysis and Proposal of Hierarchical Menu Design from the Perspective of Communicative Breakdown. The Transactions of Human Interface Society 10(3), 21-34 (2008) (in Japanese) 